\title{
Accesibilidad e Integración Digital: Elementos Clave para un Programa de Formación de Empresarios en Empoderamiento Digital
}

\author{
Raúl J. Martelo(1), Iris A. Jiménez ${ }^{(2)}$ y José D.C. Jaimes ${ }^{(3) \star}$ \\ (1) Univ.de Cartagena, Fac.de Ingeniería, Grupo de Investigación en Tecnologías de las Comunicaciones \\ e Informática (GIMATICA), Avenida el Consulado, Calle 30 No. 48-152. Cartagena, Bolívar-Colombia. \\ (2) Universidad de la Guajira, Facultad de Ciencias Básicas y Aplicadas, Grupo de Investigación BIEMARC, \\ Km 5 vía a Maicao, Guajira-Colombia. \\ (3) Universidad de Cartagena, Facultad de Enfermería, Grupo de Investigación en Medio Ambiente, \\ Alimentos y Salud (MAAS), Cra. 50 \#24-120, Cartagena, Bolívar, Colombia \\ (e-mail: jjaimesmor@yahoo.es)
}

${ }^{*}$ Autor a quien debe ser dirigida la correspondencia

Recibido Dic. 15, 2016; Aceptado Ene. 6, 2017; Versión final Mar. 13, 2017, Publicado Dic. 2017

\begin{abstract}
Resumen
El objetivo de este trabajo es proponer un Programa de Formación para el empoderamiento digital de administradores de micro, pequeñas y medianas empresas (MiPymes). Para ello, se indaga en las competencias en tecnologías de información y comunicación (TIC) que poseen los administradores de MiniPymes. Bajo una metodología de investigación-acción, se encuestaron a 250 representantes de estas empresas. Los resultados demuestran un alto índice de disponibilidad en la red y una adecuada infraestructura tecnológica, a pesar del poco empleo reportado para el uso de las TIC y la página Web, dado los bajos niveles de preparación en TIC. Se concluye que, existe una relación incongruente entre ambas dimensiones; mientras las condiciones de accesibilidad están dadas, no se aprovechan estos recursos para empoderar digitalmente a los empresarios a través de la integración de conocimientos digitales.
\end{abstract}

Palabras clave: competencias; empresarios; Mipymes; TIC; uso de la Web; empoderamiento digital

\section{Digital Accessibility and Integration: Key Elements for Entrepreneurs Training Program on Digital Empowerment}

\begin{abstract}
The objective of this work is to investigate the competences in information and communication technologies (ICT) of the administrators of micro, small and medium size enterprises (MiPymes) with the aim of proposing a Training Program for the digital empowerment of this important Colombian social sector. Under an actionresearch methodology, 250 representatives of these companies were surveyed. The results demonstrate a high availability rate in the network and an adequate technological infrastructure, despite the low employment reported for the use of ICT and the Web page, given the low levels of ICT preparation. It is concluded that there is an inconsistent relationship between both dimensions; while the conditions of accessibility are given, the advantages of these resources to digitally empower these entrepreneurs through the integration of digital knowledge are not taken into account.
\end{abstract}

Keywords: competences; enterpreneurs; Mipymes; ICT; Web use; digital empowerment 


\section{INTRODUCCIÓN}

La implementación de las Tecnologías de Información y Comunicación (TIC) ha traído como consecuencia la generación de cambios sustanciales respecto a los procedimientos adoptados por los sectores clave de la sociedad en el siglo pasado, transformándose en un factor influyente y decisivo tanto en los procesos organizacionales como en las actividades socio-productivas. Dentro de estos sectores, las MiPymes, definidas en la Ley 590 del año 2000 y la ley 905 del 2004 de la legislación Colombiana, como entidades micro, pequeñas y medianas ubicadas principalmente en comercio y en industria, las cuales reciben esta clasificación dependiendo del volumen de facturación y del número de empleados; han demostrado poseer mayores ventajas, respecto a las grandes, no solo por la flexibilidad y facilidad de adaptación a las condiciones virtuales, demandadas por las TIC, sino que también por las potencialidades que ofrecen estas tecnologías dentro de la dinámica de la revolución tecnológica (Saavedra y Tapia, 2013).

En este sentido, Botello et al. (2015) indican que la implementación e introducción de las TIC dentro de los procesos de producción es clave para el incremento de su competitividad y es por esto que las TIC son actualmente las mejores herramientas que tienen las empresas para enfrentar el contexto de la sociedad del conocimiento. Las importantes oportunidades que brindan dichas herramientas a la sociedad para su desarrollo, al estado para cumplir su rol de garante de la equidad e igualdad para sus gobernados, a la empresa como el instrumento más idóneo para desarrollar nuevos productos y servicios y a la universidad en su papel formador de las competencias requeridas, son fundamentales y han cambiado en este nuevo siglo las funciones y roles de los actores antes mencionados. Tanto el ciudadano, el Estado, la empresa y la Universidad han asumido roles compartidos y propios de su naturaleza para potenciar el uso de las TIC en sus espacios naturales con el fin de lograr orquestar beneficios para el progreso, crecimiento económico, gestión del conocimiento y tecnología, así como bienestar y desarrollo para las personas.

Esta revolución tecnológica, como toda innovación social que implica la apropiación de conocimientos específicos, ha ameritado que los gobiernos, tanto de economías desarrolladas como en desarrollo, hayan enfocado sus esfuerzos hacia la generación de ambientes adecuados que faciliten el desarrollo socioproductivo de sus países, para así poder aprovechar al máximo las potencialidades que ofrecen las TIC. Aun cuando, existe mucha información empírica sobre las tecnologías de información como mecanismo para apropiarse del conocimiento, se carece de un enfoque teórico suficientemente sistematizado y explicativo que revele el conjunto de fenómenos y factores asociados con la apropiación tecnológica y su efecto sobre la acción social (Andrade y Campo-Redondo, 2012).

Por lo tanto, los esfuerzos gubernamentales antes mencionados deben apuntar, principalmente, al empoderamiento digital de sus habitantes, creando los mecanismos necesarios de accesibilidad e integración tecnológica, que les permita utilizar adecuadamente las nuevas tecnologías de información y comunicación, hardware o software (habilidades instrumentales), y desarrollar nuevas competencias en el uso, administración y distribución de la información y del conocimiento (habilidades informacionales y estratégicas). Tanto la accesibilidad como la integración digital se consideran elementos clave para este empoderamiento ciudadano, éste último es definido por Guitert (2013) como el conjunto de fundamentos de capacitación avanzada en competencias digitales para que los individuos sean más eficientes y más críticos y, en consecuencia, desarrollen habilidades personales y profesionales que vayan más allá del simple uso de la tecnología para buscar, captar, gestionar y tratar la información, presentar y difundir los contenidos en el formato adecuado, comunicarse y colaborar en la red.

Esta definición de Guitert sobre el empoderamiento digital sitúa en primer plano la relevancia del concepto de competencia y la comprensión del mismo como una realidad que integra dimensiones, no solamente relacionadas con el acceso y uso de medios y recursos, sino también con la capacidad para descodificarlos críticamente e interactuar con ellos (Ferrés y Piscitelli, 2012). En lo que respecta a la accesibilidad digital, reconocida a partir de 2011 por la Organización para la Seguridad y la Cooperación en Europa y por la Organización de Naciones Unidas como un derecho humano fundamental para la libertad de expresión (Toudert, 2013), la misma implica proveer de conectividad a toda la población lo cual requiere sortear obstáculos de todo tipo: económicos fundamentalmente, geográficos y sin duda, políticos (Berrios-Zapata y Rojas, 2014).

Por su parte la integración digital es conceptualizada por Sánchez (2013) como el diseño propositivo de espacios de inclusión digital y de articulación de respuestas orgánicas a las asimetrías en la región. La autora se refiere a un contexto geográfico regional, donde se requiere que los países sustenten los principios de cooperación, asociatividad y reticularidad para lograr intercambio de conocimiento e información construido a través de redes y así consolidar la integración regional. Sin embargo, de acuerdo con Cáceres et al. (2015) la participación es un factor necesario pero no suficiente para empoderarse. Para estos autores, es importante que los sujetos se conviertan en ciudadanos digitales activos, pero se deben garantizar unos mínimos de implicación en acciones sustantivas para que esa participación obtenga resultados constatables a nivel individual y social. 
Ahora bien, este empoderamiento digital a través de la accesibilidad e integración digital, por parte de los ciudadanos en general y de los que forman parte de las MiPymes en particular, exige cierto grado de formación en competencias digitales para poder aprovechar las potencialidades ofrecidas por las TIC. Es aquí donde se incorporan dos factores importantes que tienen que ver con la Responsabilidad Social Empresarial (RSE) y la gestión del conocimiento dentro de este contexto. Respecto a la RSE, Daft (2014) la define como la obligación de los ejecutivos de tomar decisiones y emprender medidas que contribuyan al bienestar y a los intereses de la sociedad y de la organización. Para Herrera et al (2013), la naturaleza de la RSE se encuentra en la creencia general de que la empresa moderna tiene responsabilidades con la sociedad y que se extienden más allá de sus obligaciones con los accionistas o los inversionistas. Esto significa que también incluye aspectos internos de la empresa como por ejemplo, velar por la formación permanente de quienes forman parte de ella.

En lo que concierne a la gestión del conocimiento en el entorno empresarial, puede considerarse como un proceso de aprovechamiento de los conocimientos para lograr la innovación en procesos y productos o servicios, para una efectiva toma de decisiones y también para la adaptación de la organización al mercado (Marulanda, et al 2016). Fonseca (2012) destaca que la transferencia de conocimiento no es una entrega de un manual; el conocimiento no es algo tangible que se produce y se entrega a una persona, al contrario, requiere de una vivencia; el compartir el conocimiento implica comunicación, reproducción y conversación; el conocimiento no se transfiere, se comparte; el conocimiento implica coproducción y construcción de éste. La gestión del conocimiento tiene que ver con facilitar su distribución; conviene que la distribución se haga de abajo hacia arriba y como producto de la experiencia.

Esta aseveración de Fonseca responde a los resultados arrojados por su investigación en el Departamento de Boyacá, Colombia, los cuales evidenciaron que el $29 \%$ de los empresarios de las PYMES consultados respondió que si sabía qué significaba las TIC mientras que, un $62 \%$ respondió que no tenía idea, que escuchaban mucho hablar sobre las TIC en el sector público, mas no en el sector empresarial. En este sentido, se justifica la implementación de un Programa de formación en TIC para el empoderamiento digital del personal de las empresas, dado los acelerados cambios que provocan en el contexto empresarial, producidos por la introducción constante de estas tecnologías dentro de este sector social y que demandan un proceso de formación continua.

\section{Programa de Formación en Competencias para el empoderamiento digital}

El Programa de formación en competencias digitales para el empoderamiento digital de empresarios, tal como lo señala Gálvez (2014), deberá contar con ejes temáticos que apunten hacia objetivos, como: a) Facilitar el conocimiento de los diversos servicios y productos que ofrece la WEB; b) Dotar al personal de las herramientas cognoscitivas necesarias para que puedan identificar las potencialidades que ofrecen las TIC en lo que respecta a los cambios en la producción y en las relaciones de trabajo, donde el factor de calificación de la fuerza de trabajo pasa a ser decisivo para determinar la ubicación laboral de las personas y sus posibilidades de carrera ocupacional; c) Preparación continua del empleado en el desarrollo de competencias digitales para que sea capaz de manejar los nuevos dispositivos que vayan surgiendo a medida que la tecnología avanza; d) Capacitación continua en TIC para los gerentes encargados de manejar estas empresas, con el fin de que se conviertan en facilitadores y promotores del conocimiento tecnológico de sus subalternos y, e) Motivar el establecimiento de redes de información para la comunicación constante entre proveedores y clientes.

Vale acotar que los cinco ejes temáticos descritos no han sido seleccionados de manera fortuita ya que han sido evaluados por otros investigadores (nacionales e internacionales) y sirven de respaldo teóricometodológico al presente trabajo. Cuentan además con una clara fundamentación legal, lo cual justifica la implementación de esta propuesta de formación para el empoderamiento digital. Desde el punto de vista teórico, Gálvez (2014) muestra en su trabajo los resultados de una investigación realizada en Colombia para determinar empíricamente el impacto que, sobre la innovación de las MiPymes, tiene el uso de las tecnologías de información y comunicación (TIC). Para ello consultó a 1201 directivos de este tipo de organizaciones sobre la utilización de ocho herramientas y prácticas TIC en ellas y sobre la evolución de su desempeño innovador en productos y/o servicios, procesos, gestión y a nivel global. Los resultados descriptivos mostraron que el grado de uso de las TIC en las MiPymes colombianas es apenas aceptable.

De igual manera, Rodríguez (2012) encontró que el acceso a las TIC en Colombia es limitado y excluyente pues depende de factores netamente económicos, de infraestructura y acceso a redes. Refiere que el uso de las TIC en las personas de estrato 1 es casi nulo, las de estrato 2 y 3 es inferior, mientras que las de estrato 4,5 y 6 tienen acceso al Internet y las usan de forma permanente; esta cifra es poco alentadora para Colombia ya que los estratos 1,2 y 3 son el mayor índice de población, es claro el alto índice de brecha digital y desigualdad social. Ante estos resultados, Flórez et al. (2016) consideran necesario adelantar acciones y 
programas estatales que garanticen el acceso y el uso de las TIC por las personas menos favorecidas, un proceso más incisivo de inclusión social y laboral, que permitan una mejorar calidad de vida y participación de la ciudadanía. En este mismo orden de ideas, Mora et al., (2015) establecen que en Colombia, el 96\% de la estructura empresarial está compuesto por Mipymes, de las cuales aproximadamente el 92\% son microempresas, por lo tanto es de gran importancia el desarrollo de nuevas propuestas para el mejoramiento de la capacidad de respuesta de las Mipymes frente a las nuevas exigencias que trae la globalización y los nuevos tratados comerciales que ha venido firmando el país en los últimos años.

Dentro del ámbito internacional, existen evidencias teóricas que también fundamentan la implementación de programas de formación en TIC para este tipo de microempresarios y que sustentan las categorías de análisis seleccionadas para el desarrollo de esta investigación. Tales son los casos de los estudios realizados en España y en Costa Rica y que en este trabajo se consideran como estudios guía. El primer estudio guía es la investigación de Gómez et al. (2014) desarrollada en el Centro Internacional de Tecnologías Avanzadas (CITA) de la Universidad de Salamanca en España, denominado proyecto Timepyme. Teniendo en cuenta el contenido de los discursos producidos en las sesiones y los estudios teóricos sobre las dimensiones identificadas, los autores establecieron 7 macro categorías de análisis con sus correspondientes subcategorías, destinadas a evaluar las competencias de los microempresarios en el uso de las TIC. Dichas categorías y subcategorías sirvieron de base para la indagación desarrollada en esta investigación, las cuales giran en torno a las propuestas por el MEN (2013). Los resultados arrojados por este trabajo advirtieron demandas relacionadas directamente con la mejora del uso que ya hacen, la mayoría de empresarios, de ciertas aplicaciones en sus empresas, por lo tanto concluyen que su materialización obedece a tres ejes fundamentales: Formación, recursos y profesionales de apoyo en TIC.

El segundo estudio guía seleccionado, lo constituye la investigación de Sandí y Brenes (2013) denominada Metodología participativa evolutiva para el desarrollo de proyectos de inclusión de las tecnologías de la información y la comunicación (TIC) en la gestión empresarial de sectores productivos no formales. El objetivo de este estudio fue facilitar las herramientas tecnológicas a grupos de sectores productivos no formales ubicados en el Cantón de Pococí (Costa Rica) con el fin de permitir el acceso al conocimiento en las TIC de manera que pudieran ser integradas con sus ideas de empresarialidad para ampliar las expectativas y mejorar con ello su calidad de vida y procurar la permanencia de productores de los sectores no formales en sus actividades, por medio de la sustitución de formas empíricas con las que tradicionalmente han realizado su gestión empresarial, por formas tecnológicas acorde a sus necesidades de negocio. Esta investigación generó la inclusión de los siguientes ítems como elementos de un programa de formación para empresarios: principios básicos del computador; uso y aplicación de un sistema operativo vigente; introducción a la tecnología Internet; herramientas de ofimática; administración financiera apoyada con las TIC; herramientas multimedia y mi negocio en la Web. Ítems estos que son considerados para la indagación que se desarrolla en este trabajo.

Desde el punto de vista legal, este programa de formación se justifica porque contribuye a reforzar uno de los roles que le corresponden al entramado empresarial de Colombia el cual ha quedado consagrado y establecido en la Ley 1341 del MinTIC (2009) en su principio 1, el cual señala: "Prioridad al acceso y uso de las Tecnologías de la Información y las Comunicaciones. El Estado y, en general, todos los agentes del sector de las Tecnologías de la Información y las Comunicaciones deberán colaborar, dentro del marco de sus obligaciones, para priorizar el acceso y uso a las Tecnologías de la Información y las Comunicaciones en la producción de bienes y servicios, en condiciones no discriminatorias en la conectividad, la educación, los contenidos y la competitividad".

En cuanto a las estrategias para la capacitación y formación profesional a ser implementadas, a través del programa de formación antes descrito, las mismas deben girar en torno al desarrollo de competencias digitales. Así lo afirma Ferrari (2012) quien define este tipo de competencias como el conjunto de conocimientos, habilidades, actitudes (incluyendo por tanto las capacidades, estrategias, valores y conciencia) que se requieren cuando se utilizan las TIC y los medios digitales para realizar tareas; resolver problemas; comunicarse; gestionar la información; colaborar; crear y compartir contenidos; y construir el conocimiento de manera efectiva, eficiente, adecuada, crítica, creativa, autónoma, con flexibilidad, de manera ética, reflexiva para el trabajo, el ocio, la participación, el aprendizaje, la socialización, el consumo, y el empoderamiento.

En ese sentido, se hace necesario diagnosticar cuáles son las competencias actuales y cuáles son las competencias a desarrollar por parte de los administradores de las MIPYMES para que ejerzan un papel protagónico en accesibilidad e integración dentro del mundo empresarial, permeado por las TIC. Esto justamente se constituyó en el objetivo central de esta investigación, destinado a indagar con qué tipo de formación digital cuentan estos empresarios a fin proponer un conjunto de competencias específicas que conformen un programa de formación empresarial en TIC y que hagan posible la capacitación del 
administrador o gerente de las MIPYMES. Ello en virtud del papel que estas empresas ejercen en la sociedad, en primer lugar por su potencial empleador y, en segundo lugar, por su facilidad de incorporar en su cadena de valor, elementos diferenciadores que promuevan su competitividad.

Para llevar a cabo este diagnóstico se parte del conjunto de competencias propuestas por el Ministerio de Educación Nacional (MEN, 2013) y por los indicadores sugeridos en el Informe Global sobre Tecnologías de la Información y Comunicación (FUNDESA, 2014). De la matriz de lineamientos de competencias TIC del MEN se han adaptado y adoptado aquellas relacionadas con el entorno empresarial y que se presentan en la Tabla 1.

Tabla 1: Descripción y definición de las competencias adaptadas del MEN (2013)

\begin{tabular}{|l|l|}
\hline Competencias & \multicolumn{1}{|c|}{ Descripción } \\
\hline Tecnológica & $\begin{array}{l}\text { La capacidad para seleccionar y utilizar de forma pertinente, responsable y eficiente una variedad } \\
\text { de herramientas tecnológicas entendiendo los principios que las rigen, la forma de combinarlas } \\
\text { y su utilización en el contexto. Esto implica conocer los términos y herramientas relacionadas con } \\
\text { las TIC así como las habilidades para su manejo. }\end{array}$ \\
\hline Comunicativa & $\begin{array}{l}\text { Capacidad para expresarse, establecer contacto y relacionarse en espacios virtuales y } \\
\text { audiovisuales a través de diversos medios y con el manejo de múltiples lenguajes, de manera } \\
\text { sincrónica y asincrónica. Esta competencia demanda disponer de personal calificado para que } \\
\text { pueda establecer redes de comunicación entre proveedores y clientes. }\end{array}$ \\
\hline Investigativa & $\begin{array}{l}\text { Capacidad de utilizar las TIC para la transformación del saber y la generación de nuevos } \\
\text { conocimientos. Con esta competencia se pretende dotar al empresario de los saberes necesarios } \\
\text { para que funja como promotor de conocimientos y pueda decidir sobre relacionados con la } \\
\text { capacidad de inversión en TIC. }\end{array}$ \\
\hline De gestión & $\begin{array}{l}\text { Capacidad para utilizar las TIC en la planeación, organización, administración y evaluación de } \\
\text { manera efectiva. Este tipo de competencias facilitará la toma de decisiones en cuanto a las áreas } \\
\text { de la empresa que necesitan ser manejadas con apoyo de las TIC. }\end{array}$ \\
\hline
\end{tabular}

Respecto a los indicadores sugeridos por FUNDESA (2014), los cuales son utilizados para medir el desarrollo alcanzado por los gobiernos de la región, en cuanto a resultados de integración y accesibilidad, se consideraron para efectos de esta investigación, los siguientes:

Índice de disponibilidad de red: El término disponibilidad de red hace referencia a la probabilidad de que el servicio de Internet funcione adecuadamente en cualquier momento.

Regulación: Efectividad de los legisladores, leyes relacionadas con las TIC, independencia judicial, eficiencia del sistema legal para resolver disputas, eficiencia del sistema legal hacer velar las regulaciones, protección a la propiedad intelectual, software pirata, número de procedimientos para hacer cumplir un contrato y número de días para hacer cumplir un contrato. Todo lo cual tiene que ver con el uso de la WEB.

Infraestructura: Electricidad producida, cobertura de red móvil, Internet de banda ancha, servidores seguros de Internet, acceso a contenido digital. Dentro de este ítem el Informe de FUNDESA (2014) señala como infraestructura el aporte de los gobiernos para crear facilidades a las empresas, al promover y regular los servicios de banda ancha, servicios de electricidad, pero cada empresa debe crear su estructura de servicios adecuada a su negocio y con el nivel de modernización exigido por sus grupos de interés.

Calidad de las instituciones de investigación: Nivel de absorción de la tecnología por parte de las empresas, capacidad de innovación, número de patentes, uso de Internet por parte de las empresas, nivel de capacitación del staff.

Niveles de preparación individual: Porcentaje de matriculación en educación secundaria, educación universitaria, porcentaje de alfabetismo.

Nivel de uso individual de TIC: Número de suscriptores de teléfonos móviles, usuarios de Internet, casas con computadoras personales, casas con acceso a Internet, suscriptores de Internet de banda ancha, suscriptores de Internet móvil, uso de redes sociales.

Tanto las competencias seleccionadas como los indicadores antes descritos contribuyeron a la planificación indagatoria de elementos claves en gerentes y empleados de MIPYMES en el Departamento de La Guajira. 
Esto con apoyo de la Universidad de La Guajira (UNIGUAJIRA) la cual cuenta con una línea de investigación relacionada con las TIC en el entorno empresarial. De esta manera se justifica el desarrollo del presente estudio no solo porque ayudará a consolidar esta línea de investigación sino porque también permitirá corroborar los hallazgos realizados por las investigaciones previas realizadas en el contexto microempresarial, que evidencian el casi total desconocimiento de las TIC (Fonseca, 2012), el nivel apenas aceptable de su uso y manejo (Gálvez, 2014) y las limitaciones en cuanto a la accesibilidad (Rodríguez, 2012). Dificultades que necesitan ser investigadas, dada la importancia de estas estructuras empresariales (reportada por Mora et al., 2015) para la economía y generación de empleo en el ámbito nacional.

\section{MATERIALES Y MÉTODOS}

Para el alcance del objetivo propuesto se adoptó una metodología que comparte rasgos de los enfoques de investigación cualitativos y cuantitativos. El rasgo cualitativo se evidencia en el tipo de investigación-acción ya que, se realizó un análisis-diagnóstico de la situación problemática en la práctica, se recolectó la información empírica sobre la misma y se formuló un programa de acción para resolver el problema (Albert, 2007). Dicho programa parte del análisis FODA realizado a los resultados obtenidos y consiste en la construcción de una matriz a partir de la identificación de un listado de factores internos (fortalezas y debilidades) y externos (oportunidades y amenazas) que influyen en el desempeño cualquier organización (Nikulin y Becker, 2015).

El rasgo cuantitativo se manifiesta en el conteo de los datos recolectados, asumiendo una metodología descriptiva, no experimental, de campo y de corte transversal o transaccional dado que, las dimensiones de la variable del empoderamiento digital se midieron en un único momento (Hernández et al 2011). La revisión documental de dichas dimensiones permitió describir y analizar las respuestas emitidas por la muestra, en el mismo campo o contexto donde se realizó la investigación, es decir en el Departamento de la Guajira.

\section{Población y muestra}

La población objeto de estudio estuvo conformada por dos representantes de las 173 MIPYMES con los cuales la Universidad de la Guajira ha establecido convenio, la mayoría pertenecientes al ramo de operaciones de manufactura, servicios y mercadeo. De esta población se seleccionó un total de 250 representantes, mediante la aplicación del censo poblacional con el fin de cubrir el total de la misma. Dicha selección se realizó de manera estratégica, tomando como criterios de inclusión: que formaran parte de la gerencia de la empresa y su voluntad y disponibilidad para el suministro de los datos.

\section{Procedimiento para la recolección de datos}

Para recoger los datos de la muestra seleccionada se diseñó un cuestionario, destinado a medir las dos dimensiones de la variable: empoderamiento digital, a saber, accesibilidad e integración digital. Las categorías de análisis estuvieron constituidas por los índices sugeridos por FUNDESA (2014) mientras que, los indicadores por las competencias establecidas en los lineamientos del MEN (2013). La tabla 2, muestra esta distribución.

Tabla 2: Categorías e indicadores evaluados

\begin{tabular}{|c|l|l|l|}
\hline Variable & Dimensiones & \multicolumn{1}{|c|}{ Categorías de análisis } & \multicolumn{1}{c|}{ Indicadores: Competencias } \\
\hline \multirow{5}{*}{$\begin{array}{c}\text { Empoderamiento } \\
\text { digital }\end{array}$} & $\begin{array}{c}\text { Accesibilidad } \\
\text { digital }\end{array}$ & $\begin{array}{l}\text { Índice de disponibilidad de red } \\
\text { Infraestructura } \\
\text { TIC usadas en la empresa } \\
\text { Uso de la WEB }\end{array}$ & $\begin{array}{l}\text { Investigativa: } \\
\text { Capacidad de inversión en TIC } \\
\text { De Gestión: } \\
\text { Área de la empresa manejada con las TIC }\end{array}$ \\
\cline { 2 - 4 } & $\begin{array}{l}\text { Integración } \\
\text { digital }\end{array}$ & $\begin{array}{l}\text { Niveles de preparación } \\
\text { individual } \\
\text { Niveles de preparación del } \\
\text { personal de la empresa } \\
\text { Nivel de uso individual de las } \\
\text { TIC }\end{array}$ & $\begin{array}{l}\text { Tecnológicas: } \\
\text { Conocimiento del concepto } \\
\text { Uso del concepto o herramienta digital } \\
\text { Habilidad para su manejo } \\
\text { Comunicativas: } \\
\text { Disponibilidad de personal calificado }\end{array}$ \\
\hline
\end{tabular}

Para cada una de estas categorías se utilizaron ítems relacionados con sus temáticas, para un total de 42 preguntas, las cuales se presentan en la Tabla 3. La escala de frecuencias utilizada contó con opciones de respuesta cerradas que variaron entre: Siempre (5), Casi Siempre (4), Algunas Veces (3), Rara vez (2) y Nunca (1). Vale acotar que este cuestionario fue sometido a las pruebas de validez y confiabilidad pertinentes, por parte de expertos metodológicos y de contenido, arrojando como resultado 0.91 , lo cual convierte en un instrumento confiable (Hernández et al 2011). 
Tabla 3: Cuestionario aplicado a la muestra

\begin{tabular}{|c|c|}
\hline$N^{\circ}$ & Preguntas relacionadas con los indicadores \\
\hline 1. & ¿Ha usado el termino Disponibilidad de Red? \\
\hline 2. & ¿Ha usado el término infraestructura para uso de las TIC? \\
\hline 3. & ¿Ha recibido información sobre la preparación individual para manejo de las TIC? \\
\hline 4. & ¿Existe preparación sobre TIC para el personal de la empresa? \\
\hline 5. & ¿Se utilizan las TIC a nivel individual por parte de los empleados y gerentes? \\
\hline 6. & ¿Existen diferentes tecnologías de información y comunicación usadas en la empresa? \\
\hline 7. & ¿En la empresa se cuenta con un servicio de Internet accesible? \\
\hline 8. & ¿Su empresa posee equipos y servicios útiles para las TIC? \\
\hline 9. & ¿Realiza actividades de capacitación para su personal? \\
\hline 10. & ¿Se ofrecen actividades que contribuyen con la preparación de equipos de trabajo en las TIC? \\
\hline 11. & ¿En el área administrativa, todo el personal tiene competencias en TIC? \\
\hline 12. & ¿Posee la empresa inventarios de las tecnologías usadas en cada área de su empresa? \\
\hline 13. & ¿Se usa la Web para realizar algún trámite de su empresa? \\
\hline 14. & ¿El personal de su empresa tiene competencias para usar Internet? \\
\hline 15. & ¿El personal de su empresa tiene habilidades para usar las TIC? \\
\hline 16. & ¿Ha identificado algún personal en su empresa que tenga habilidades para enseñar a otros? \\
\hline 17. & ¿La empresa tiene un plan de capacitación en TIC para su personal? \\
\hline 18. & ¿Existe apoyo de algún personal para capacitar a otros sobre uso de las TIC? \\
\hline 19. & ¿Tiene la empresa un inventario de personas que manejan esas tecnologías? \\
\hline 20. & ¿Se emplea la Web para las operaciones de producción? \\
\hline 21. & ¿Cuándo contrata personal exige habilidades en el manejo de las TIC? \\
\hline 22. & ¿El personal recibe capacitación sobre uso de las TIC? \\
\hline 23. & ¿El personal de su empresa exhibe conocimiento sobre el uso de las TIC? \\
\hline 24. & ¿Contrata la empresa asesoría para establecer las necesidades de entrenamiento? \\
\hline 25. & ¿La empresa puede invertir en cursos de preparación en el uso individual de TIC para la comunidad? \\
\hline 26. & ¿La empresa acude al mercado laboral para buscar personal calificado en TIC? \\
\hline 27. & ¿El personal de la empresa exhibe dominio para el uso de la Web en su trabajo? \\
\hline 28. & ¿Tiene la empresa capacidad para invertir en TIC? \\
\hline 29. & ¿Tiene un presupuesto para compra de tecnologías de información y comunicación? \\
\hline 30. & ¿Financia actividades de capacitación en TIC para su personal? \\
\hline 31. & ¿El departamento de recursos humanos ejerce un importante papel en la capacitación del personal? \\
\hline 32. & ¿Se usan teléfonos digitales entre el personal de la empresa? \\
\hline 33. & ¿Hay disposición en la junta directiva para invertir en TIC en nuevas áreas de su empresa? \\
\hline 34. & ¿Se recurre a personal externo para el uso de la Web en su negocio? \\
\hline 35. & ¿Usa las TIC para el área administrativa? \\
\hline 36. & ¿Usa las Tic para mercadeo y ventas? \\
\hline 37. & ¿En el área operativa ha contratado personal para el manejo de las TIC? \\
\hline 38. & ¿Existen planes para capacitar al personal del área operativa o de producción en TIC? \\
\hline 39. & ¿Hay disponibilidad de computadoras en casa? \\
\hline 40. & ¿Existe un plan para cubrir toda la gama de actividades de su empresa con apoyo de las TIC? \\
\hline 41. & ¿Tiene la empresa especial interés para invertir en TIC para nuevas áreas en su empresa? \\
\hline 42. & ¿Existe un plan para cubrir toda la gama de actividades de su empresa con apoyo de las TIC? \\
\hline
\end{tabular}

Análisis de la información

Para el análisis de la información recolectada, producto de la aplicación del cuestionario a la población seleccionada, se procedió a la tabulación de las respuestas emitidas por cada uno de los participantes de la muestra. Para ello, se siguió un tratamiento estadístico de tipo descriptivo, utilizando distribuciones de 
frecuencias absolutas y relativas (porcentajes) y medidas de tendencia central para cada ítem e indicador medido. Estos resultados fueron analizados y expresados en tablas de frecuencia, que facilitaron su interpretación a fin de dar cumplimiento al propósito del estudio.

\section{RESULTADOS Y DISCUSIÓN}

Este apartado ha sido dividido en siete secciones, las cuales abordan los resultados generados por las seis categorías sometidas a análisis, constituidas por: Índice de disponibilidad de red, Infraestructura, Niveles de preparación individual, Niveles de preparación del personal de la empresa, Nivel de uso individual de las TIC, TIC usada en la empresa y Uso de la WEB. En este abordaje se incluyen los estadísticos resumen de cada bloque de preguntas formuladas para cada categoría. La séptima sección se dedica a presentar la propuesta del Programa de Formación con los ejes temáticos, derivados tanto de los resultados obtenidos como del análisis FODA practicado a los mismos.

\section{Índice de Disponibilidad en Red}

Respecto a si la empresa conoce el índice de disponibilidad de red y si es útil ese índice para su proceso, la Tabla 4 muestra los resultados correspondientes a esta disponibilidad. Luego, si se hace un balance, un $70,29 \%$ establece que la empresa utiliza y conoce dicho índice.

Tabla 4: Resultados sobre el Índice disponibilidad de red

\begin{tabular}{|c|c|c|c|c|c|c|c|c|c|}
\hline Preguntas & Tipo & $S(5)$ & $C S(4)$ & $A V(3)$ & $R V(2)$ & $N(1)$ & Total & Media & $\begin{array}{c}\text { Desv. } \\
\text { estándar }\end{array}$ \\
\hline $\begin{array}{c}1,8,15,22,29, \\
36\end{array}$ & FA & 57 & 40 & 23 & 16 & 2 & 138 & \multirow{2}{*}{4,0} & 1,09 \\
\cline { 2 - 8 } & $\%$ & 41,30 & 28,99 & 16,67 & 11,59 & 1,45 & 100 & & \\
\hline
\end{tabular}

Este hallazgo revela la existencia de un servicio de Internet; que el personal cuenta con competencias para su uso; que se invierte para el servicio y por lo menos el área administrativa lo maneja. Este resultado coincide con lo señalado por Saavedra y Tapia (2013) en relación a la importancia de contar con disponibilidad de red para lograr conectividad y servicios para la empresa.

\section{Infraestructura Tecnológica}

Las preguntas relacionadas con la dotación de la empresa en infraestructura tecnológica, arrojaron como resultado general que el $82,61 \%$ de los encuestados indicaron que la empresa cuenta con la tecnología necesaria de información y comunicación para sus procesos. Estos resultados se presentan en la Tabla 5.

Tabla 5: Resultados sobre la Infraestructura Tecnológica

\begin{tabular}{|c|c|c|c|c|c|c|c|c|c|}
\hline Preguntas & Tipo & $S(5)$ & $C S(4)$ & $A V(3)$ & $R V(2)$ & $N(1)$ & Total & Media & $\begin{array}{c}\text { Desv. } \\
\text { estándar }\end{array}$ \\
\hline \multirow{2}{*}{$2,9,16,23,30,37$} & FA & 85 & 29 & 15 & 6 & 3 & 138 & \multirow{2}{*}{3,9} & 0,83 \\
\cline { 2 - 10 } & $\%$ & 61,59 & 21,01 & 10,87 & 4,35 & 2,17 & 100 & \\
\hline
\end{tabular}

Los resultados de la Tabla 5 cubren las dificultades referidas a: la ausencia de capacitación para manejar toda la infraestructura, la escasa capacitación del personal y el dueño para este propósito, la existencia de presupuesto para la compra y el uso restringido de correos electrónicos para los clientes en el área de facturación. Al superar estas dificultades se infiere que las MIPYMES en las cuales labora la muestra encuestada se han preocupado por crear una estructura de servicios adecuada a su negocio y con el nivel de modernización exigido por sus grupos de interés tal como lo señala el FUNDESA (2014).

\section{Niveles de preparación individual}

En relación a la preparación individual de los gerentes en TIC para el tratamiento de los diferentes rubros señalados, si se hace un balance negativo, un $82,61 \%$ establece que los gerentes no tienen el nivel de preparación necesario para usar las potencialidades de las TIC. Estos resultados se resumen en la Tabla 6.

Tabla 6: Resultados sobre los Niveles de preparación individual

\begin{tabular}{|c|c|c|c|c|c|c|c|c|c|}
\hline Preguntas & Tipo & $S(5)$ & $C S(4)$ & $A V(3)$ & $R V(2)$ & $N(1)$ & Total & Media & $\begin{array}{c}\text { Desviación } \\
\text { estándar }\end{array}$ \\
\hline \multirow{2}{*}{$\begin{array}{c}3,10,17,24,31 \text { y } \\
38\end{array}$} & FA & 3 & 6 & 15 & 29 & 85 & 138 & \multirow{2}{*}{3,9} & \multirow{2}{*}{0,83} \\
\cline { 2 - 9 } & $\%$ & 2,17 & 4,35 & 10,87 & 21,01 & 61,59 & 100 & & \\
\hline
\end{tabular}


Vale aclarar que dentro de estos hallazgos también se encuentran, actividades de capacitación hacia el empleado, búsqueda de acompañamiento interno para capacitación y la contratación de personal para las TIC en el área de producción. Los resultados arrojados para este nivel de preparación contrastan con los ventajas que tienen aquellos gerentes con competencias en TIC, que tal como las define Ferrari (2012) son habilidades, actitudes que se requieren cuando se utilizan las TIC y los medios digitales para realizar tareas; resolver problemas; comunicarse; gestionar la información; colaborar; crear y compartir contenidos; y construir el conocimiento de manera efectiva, eficiente, adecuada, crítica, creativa, autónoma, con flexibilidad, de manera ética, reflexiva para el trabajo, el ocio, la participación, el aprendizaje, la socialización, el consumo, y el empoderamiento.

\section{Niveles de preparación del personal de la empresa}

En virtud de las respuestas, tal como se muestra en la Tabla 7, el balance indica que $45,65 \%$ admite que la organización contrata personal con experiencia y competencias en TIC, sin embargo, es importante resaltar que existe un $30,43 \%$ con dudas sobre la experiencia de dicho personal. Este resultado también comprende interrogantes sobre: la preparación de equipos de trabajo en las TIC, plan de capacitación en TIC para su personal, asesoría para establecer las necesidades de entrenamiento, papel del departamento de recursos humanos en la capacitación y planes para capacitar personal para el área operativa o de producción.

Tabla 7: Resultados sobre los Niveles de preparación del personal de la empresa

\begin{tabular}{|c|c|c|c|c|c|c|c|c|c|}
\hline Preguntas & Tipo & $S(5)$ & CS (4) & $A V(3)$ & $R V(2)$ & $N(1)$ & Total & Media & $\begin{array}{c}\text { Desv. } \\
\text { estándar }\end{array}$ \\
\hline $\begin{array}{c}4,11,18,25,32 \text { y } \\
39\end{array}$ & FA & 8 & 27 & 42 & 55 & 6 & 138 & \multirow{2}{*}{3,7} & \multirow{2}{*}{0,98} \\
\cline { 2 - 11 } & $\%$ & 5,80 & 39,86 & 30,43 & 19,57 & 4,35 & 100 & & \\
\hline
\end{tabular}

Esta diferencia en cuanto a preparación, demuestra que los mecanismos de integración digital implementados por la empresa no están llegando a todos los empleados de manera equitativa y que por lo tanto, pudiera estar ocurriendo lo que Cáceres (2015) establece para estas desigualdades, es decir que no basta con la participación en los procesos mediados por las TIC para estar empoderado digitalmente, sino que también se deben garantizar unos mínimos de implicación en acciones sustantivas para que esa participación obtenga resultados constatables a nivel individual y social.

\section{Nivel de uso individual de las TIC}

En cuanto a la utilización de las TIC a nivel individual por parte de los empleados y gerentes, un $58,70 \%$ de la organización expresó que no hay uso suficiente a nivel individual de las TIC por parte de los empleados de la empresa. De la medición de esta categoría también se encontraron los siguientes aspectos: en el área administrativa, no todo el personal tiene competencias en TIC, no existe apoyo de algún personal para capacitar a otros sobre uso de las TIC, la empresa puede invertir en cursos de preparación en el uso individual de TIC para la comunidad inmediata pero no lo hace, poco uso de teléfonos así como también escasa disponibilidad de computadoras en casa. La Tabla 8 ilustra estos resultados.

Tabla 8: Resultados sobre el Nivel de uso individual de TIC

\begin{tabular}{|c|c|c|c|c|c|c|c|c|c|}
\hline Preguntas & Tipo & $S(5)$ & $C S(4)$ & $A V(3)$ & $R V(2)$ & $N(1)$ & Total & Media & $\begin{array}{c}\text { Desviación } \\
\text { estándar }\end{array}$ \\
\hline \multirow{2}{*}{$5,12,19,26,33,40$} & FA & 3 & 16 & 38 & 45 & 36 & 138 & \multirow{2}{*}{4,0} & \multirow{2}{*}{1,09} \\
\cline { 2 - 8 } & $\%$ & 2,17 & 11,59 & 27,54 & 32,61 & 26,09 & 100 & & \\
\hline
\end{tabular}

Dichos resultados evidencian la carencia de una infraestructura tecnológica adecuada y el bajo conocimiento de uso que existe, a nivel de hogares. Los datos recolectados coinciden con los hallazgos de Rodríguez (2012) quien encontró que el acceso a las TIC en Colombia es limitado y excluyente pues, depende de factores netamente económicos, de infraestructura y acceso a redes. De igual manera, concuerdan con los resultados descriptivos reportados por Gálvez (2014), demostrando que el grado de uso de las TIC en las MIPYME colombianas es apenas aceptable.

\section{TIC usadas en la empresa}

En relación con este uso y en virtud de las respuestas, el balance indica que un $68,84 \%$ admite que la empresa usa diferentes TIC en su área administrativa, esta dimensión cubrió también aspectos como: falta de inventario de las TIC instaladas o usadas en su empresa, falta de inventario de personas que manejan esas tecnologías y han recibido capacitación, no se acude el mercado laboral para buscar personal calificado en TIC, 
disposición en la junta directiva de invertir en TIC para nuevas áreas en su empresa y la poca frecuencia de uso de las TIC en todas las operaciones de la empresa. La Tabla 9 describe estos hallazgos.

Tabla 9: Resultados sobre las TIC usadas en la empresa

\begin{tabular}{|c|c|c|c|c|c|c|c|c|c|}
\hline Preguntas & Tipo & $S(5)$ & $C S(4)$ & $A V(3)$ & $R V(2)$ & $N(1)$ & Total & Media & $\begin{array}{c}\text { Desviación } \\
\text { estándar }\end{array}$ \\
\hline \multirow{2}{*}{$6,13,20,27,34,41$} & FA & 33 & 62 & 36 & 6 & 1 & 42 & \multirow{2}{*}{4,0} & 1,09 \\
\cline { 2 - 9 } & $\%$ & 23,91 & 44,93 & 25,09 & 4,35 & 0,72 & 100 & \\
\hline
\end{tabular}

La ubicación, en casi siempre, de la mayoría de la muestra encuestada $(44,93 \%)$ puede interpretarse como la poca atención dada a unos de los principios establecidos en la Ley 1341 del MinTIC (2009), el cual señala: "Prioridad al acceso y uso de las Tecnologías de la Información y las Comunicaciones en condiciones no discriminatorias en la conectividad, la educación, los contenidos y la competitividad. Así mismo, se evidencia que las obligaciones de la RSE de tomar decisiones y emprender medidas que contribuyan al bienestar y a los intereses de la sociedad y de la organización no se están cumpliendo a cabalidad. Tal como lo señalan Herrera et al (2013), la naturaleza de la RSE debe extenderse más allá de sus obligaciones con los accionistas o los inversionistas. Esto significa que también incluye aspectos internos de la empresa como por ejemplo, velar por la formación permanente de quienes forman parte de ella. En este sentido, vale acotar la afirmación que hacen Botello et al (2015) en cuanto a la implementación e introducción de las TIC dentro de los procesos de producción como elementos clave para el incremento de su competitividad y como las mejores herramientas que tienen las empresas para enfrentar el contexto de la sociedad del conocimiento.

Uso de la WEB

En lo que concierne al uso de la WEB por parte de la muestra encuestada, el balance indica que el $61,59 \%$ expresa que la organización no usa la Web como plataforma de mercadeo o comercio electrónico. También se encontraron en esta dimensión los siguientes resultados: Rara vez se usa la Web para realizar algún trámite de su empresa, tampoco se emplea la Web para las operaciones de producción, el personal de la empresa exhibe poco dominio para el uso de la Web en su trabajo, no se ha recurrido a personal externo para el uso de la Web en su negocio, se cree que es costosa la inversión para el uso de la Web en sus operaciones de distribución y venta y no se ha realizado ningún plan para cubrir toda la gama de actividades de su empresa con apoyo de las TIC.

Tabla 10: Resultados sobre el Uso de la Web

\begin{tabular}{|c|c|c|c|c|c|c|c|c|c|}
\hline Preguntas & Tipo & $S(5)$ & $C S(4)$ & $A V(3)$ & $R V(2)$ & $N(1)$ & Total & Media & $\begin{array}{c}\text { Desviación } \\
\text { estándar }\end{array}$ \\
\hline \multirow{2}{*}{$7,13,21,28,35$ y 42} & FA & 5 & 24 & 24 & 58 & 27 & 138 & 2,8 & \multirow{2}{*}{1,23} \\
\cline { 2 - 9 } & $\%$ & 3,62 & 17,39 & 17,39 & 42,03 & 19,57 & 100 & 2,8 & 1,23 \\
\hline
\end{tabular}

Este bajo porcentaje reportado en el uso de plataformas Web por parte de estos empresarios, indica el desaprovechamiento de las potencialidades que ofrecen las TIC y por ende la falta de empoderamiento digital de esta población, al no emplear los mecanismos de accesibilidad e integración digital que demanda la actual sociedad del conocimiento. Esto pudiera deberse a los factores que mencionan Berrios-Zapata y Rojas (2014) relacionados con los obstáculos económicos fundamentalmente, geográficos y sin duda, políticos que tienen que de seguro tienen que sortear estas empresas para lograr tal empoderamiento. En tal sentido se infiere que hace falta el diseño propositivo de espacios de inclusión digital y de articulación de respuestas orgánicas a las asimetrías en la región del cual hace mención Sánchez (2013) y que son requeridos para sustentar los principios de cooperación, asociatividad y reticularidad, destinados a lograr intercambio de conocimiento e información a través de redes para así consolidar la integración regional.

En términos generales, puede afirmarse que estos resultados corroboran la situación problemática planteada al inicio de esta investigación y que fue reportada por autores como Fonseca (2012), Gálvez (2014), Rodríguez (2012) y Mora et al. (2015), quienes destacan las limitaciones de accesibilidad e integración digital para el empoderamiento digital por parte de las MiPymes en el contexto empresarial. De allí la necesidad de implementar acciones que se dediquen a capacitar a estos empresarios en el uso y manejo de las TIC, a través de un Programa de Formación como el sugerido en este trabajo.

\section{Análisis FODA y propuesta del Programa de Formación para el empoderamiento digital}

A partir de los resultados descritos, se construye la matriz de análisis FODA con los factores positivos o fortalezas y factores débiles o no consolidados en el manejo de las TIC en las operaciones de manufactura, servicios y mercadeo de las empresas encuestadas. Este análisis se resume en la Tabla 11. 
Tabla 11: Fortalezas y Debilidades extraídas de los Resultados

\begin{tabular}{|l|l|}
\hline \multicolumn{1}{|c|}{ Fortalezas } & \multicolumn{1}{c|}{ Debilidades } \\
\hline $\begin{array}{l}\text { Conocimiento y habilidad para el uso de } \\
\text { Internet }\end{array}$ & Uso reducido de las bondades de las TIC \\
\hline $\begin{array}{l}\text { Disponibilidad de infraestructura tecnológica } \\
\text { para uso de las TIC (correo, pagina Web, } \\
\text { redes sociales, cuenta de Internet, software } \\
\text { actualizado para área administrativa) }\end{array}$ & $\begin{array}{l}\text { No poseen experiencia ni habilidad en comercio electrónico. } \\
\text { No usan la Web para mercadeo } \\
\text { No hacen retroalimentación con el cliente. }\end{array}$ \\
\cline { 2 - 2 } $\begin{array}{l}\text { Poco uso de las TIC a nivel individual de miembros de la } \\
\text { empresa }\end{array}$ \\
\hline \multirow{2}{*}{$\begin{array}{l}\text { Experiencia y conocimiento para Uso de Tic } \\
\text { en procesos operativos de la empresa }\end{array}$} & $\begin{array}{l}\text { No se orienta ni se explota el potencial del empleado preparado } \\
\text { en TIC }\end{array}$ \\
\cline { 2 - 2 } & $\begin{array}{l}\text { Uso reducido en las áreas de búsqueda de nuevos proveedores. } \\
\text { No realizan cursos online para el área técnica. }\end{array}$ \\
\hline
\end{tabular}

Con base en este FODA y considerando los hallazgos reportados por la muestra se proponen los elementos principales, requeridos en el contenido de un programa de formación para el empoderamiento digital de emprendedores, gerentes y dueños de MiPymes en del Departamento de la Guajira que se encuentran vinculados a la Universidad de la Guajira. Estos elementos o ejes temáticos se muestran en la Figura 1.
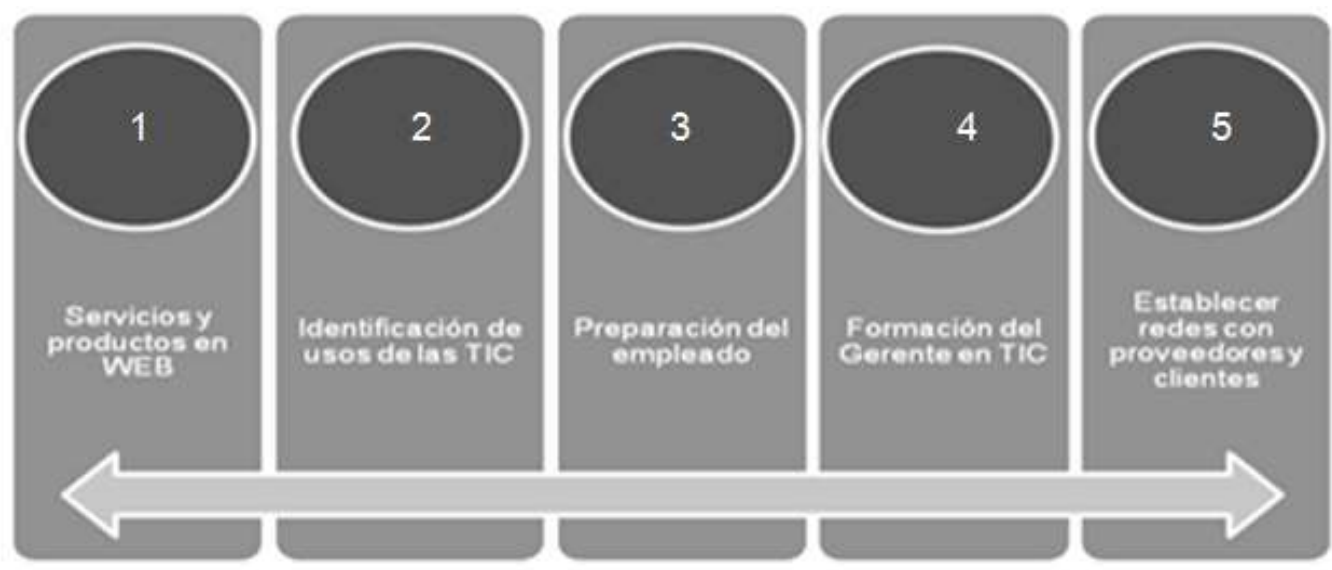

Fig. 1: Propuesta de ejes temáticos para el programa de formación de Gerentes de MiYPYME

Como puede observarse en la figura, estos ejes temáticos parten, desde el conocimiento de los servicios y productos que ofrece la WEB, pasan por la identificación del uso de las TIC y la preparación en estas tecnologías para los empleados y gerentes, hasta llegar a dotar a este personal de las herramientas cognoscitivas necesarias para que puedan establecer redes de información con los proveedores y clientes. Tales ejes fueron considerados también por Sandí y Brenes (2013) y pretenden facilitar la gestión del conocimiento en TIC bajo una metodología participativa. En fin, las bases teóricas que fundamentan estos ejes temáticos, discutidas en la introducción de este trabajo, ofrecen un panorama conceptual para su implementación cuyo objetivo primordial debe tener como norte empoderar digitalmente a los empresarios de esta MiPymes a través de la accesibilidad e integración digital, consideradas como elementos clave para dicho empoderamiento.

\section{CONCLUSIONES}

Las conclusiones generadas a través de esta investigación se basan en los resultados reportados por la muestra encuestada para cada una de las seis categorías analizadas, derivadas de la accesibilidad e integración digital como dimensiones de la variable empoderamiento digital. En consecuencia se concluye lo siguiente:

Respecto al índice de disponibilidad de red, en estas empresas existe conocimiento sobre este índice, cuentan con la infraestructura necesaria, servicio de Internet y algunos desarrollan competencias para su uso. Esto no ocurre con la plataforma en la cual están dispuestos a invertir pero, tanto los gerentes como los empleados no poseen conocimientos sobre las ventajas que esta infraestructura ofrece, dada la escasa capacitación y formación reportada en esta categoría. 
En relación con los bajos niveles de preparación individual, nivel de preparación del personal de la empresa y uso de las TIC, se demostró los pocos esfuerzos realizados por los empresarios para obtener este tipo de formación, la insuficiente disposición de las MiPymes para la formación de este personal en competencias digitales y para crear mecanismos de accesibilidad e integración digital tanto internamente como para sus clientes a través del uso de las TIC. Esta falta de disposición se evidenció también en el empleo casi nulo de la página WEB para establecer relaciones de mercadeo, ventas y servicios al cliente dado que, no cuentan con las competencias digitales internas para su manejo y no tienen intenciones de solicitar asesoría externa para la utilización de este recurso tecnológico.

Existe una relación incongruente entre las dimensiones estudiadas ya que, mientras están dadas las condiciones de accesibilidad no se aprovechan estos recursos para empoderar digitalmente a los empresarios a través de la integración de conocimientos digitales.

\section{REFERENCIAS}

Albert, M., La investigación Educativa. Claves Teóricas. 2ª Ed., McGraw-Hill, Interamericana de España, Madrid, España (2007)

Andrade, J. y M. Campo-Redondo, Apropiación social de las tecnologías de información: políticas públicas para la participación ciudadana, Quórum Académico, (9)1, 52-68 (2012)

Berrio-Zapata, C. y H. Rojas, La Brecha Digital Universitaria: La Apropiación de las TIC en Estudiantes de Educación Superior en Bogotá, Revista Científica de Educomunicación, Comunicar, 43 (XXII),133-142 (2014)

Botello, H., Pedraza, A. y O. Contreras, Análisis Empresarial de la influencia de las TIC en el desempeño de las empresas de servicios en Colombia, Revista Virtual Universidad Católica del Norte, (45) 3-15 (2015)

Cáceres, M., y otros tres autores, Hacia la construcción de una ciudadanía digital. Nuevos modelos de participación y empoderamiento a través de Internet, Prisma Social, 15(2), 643-683 (2015)

Daft, R., Teoría y Diseño Organizacional, 11aㅡ Ed., International Thomson, México (2014)

Ferrari, A., Digital Competence in Practice: An Analysis of Frameworks. Luxembourg: Publications Office of the European Union. ISBN 978-92-79-25093-4 (pdf). ISSN 1831-9424 (online). Doi:10.2791/82116 (2012). Disponible en: https://goo.gl/kRvfui. Acceso 16 de agosto (2016)

Ferrés, J. y A. Piscitelli, La competencia mediática: propuesta articulada de dimensiones e indicadores, Comunicar, (38) 75-82 (2012)

Flórez, D., C. Ramírez y S. Ramírez, Las Tic como herramientas de inclusión social, Revista 3C TIC, (16) 5, 54-6 (2016)

Fonseca, D., La Prospectiva y el Conocimiento de las TICS en las Pymes del Departamento de Boyacá, Colombia, Pensamiento y Gestión, (33) 182-202 (2012)

Fundación para el Desarrollo de Guatemala (FUNDESA), Informe global sobre Tecnologías de la Información y Comunicación. Recompensas y Riesgos de grandes datos (2014). Disponible en: https://goo.gl/zxtw1y, Acceso 16 de agosto (2016)

Gálvez, E., Tecnologías de Información y Comunicación, e Innovación en las MIPYMES de Colombia, Cuadernos de Administración, (30) 51, 71-79 (2014)

Guitert, M., Tiempo de empoderamiento digital (2013). Disponible en: https://goo.gl/D8NbeN, Acceso 14 de abril (2013)

Gómez, M. y otros tres autores, Percepciones de los empresarios de Pymes rurales sobre la integración de las TIC, Revista Ibérica de Sistemas de Tecnologías de Información, (2) 71-84 (2014)

Hernández, R., Fernández, C., Baptista, P., Metodología de la investigación. 5aㅡ Ed., 613. McGraw-Hill Interamericana, México, D.F., México (2011) 
Herrera, M., Larran, J. y B. Martínez, Relación entre responsabilidad social y performance en las pequeñas y medianas empresas, Cuadernos de Gestión, (13) 2, 39-65 (2013)

Marulanda, C., M. López y F. López, La Cultura Organizacional y las Competencias para la Gestión del Conocimiento en las Pequeñas y Medianas Empresas (PYMEs) de Colombia, Información Tecnológica 27(6), 3-10 (2016)

Ministerio de Educación Nacional, Competencias TIC para el desarrollo profesional docente. Bogotá: MEN (2013)

Ministerio de Tecnología de Información y Comunicación (MinTIC), Ley 1341 de 2009. Disponible en https://goo.gl/qu8qGi. Acceso 16 de agosto 2016 (2009)

Mora, E., M. Vera y Z. Melgarejo, Planificación estratégica y niveles de competitividad de las Mipymes del sector comercio en Bogotá, Estudios Gerenciales, (31) 134, 79-87 (2015)

Nikulin, C. y G. Becker, Una metodología Sistémica y creativa para la gestión estratégica: Caso de Estudio Región de Atacama-Chile, J. Technol. Manag. Innov., (10) 1 (2015)

Rodríguez, P., Límites y posibilidades de las TIC en la inclusión social, en: Tecnologías de la información para la inclusión social: una apuesta por la diversidad, por E. Vallejo y otros cinco autores, 40-47. Colombia Digital, Boogtá, D.C. Colombia (2012)

Saavedra, M. y B. Tapia, El uso de las tecnologías de información y comunicación TIC en las micro, pequeñas y medianas empresas (MIPyME) industriales mexicanas, Enl@ce: Revista Venezolana de Información, Tecnología y Conocimiento, 10(1), 85-104 (2013)

Sánchez, S., DebaTIC: El valor de las Tecnologías de Información y Comunicación (TIC) en la Integración Regional. SELA, Caracas (2013)

Sandí, J. y C. Brenes, Metodología participativa evolutiva para el desarrollo de proyectos de inclusión de las tecnologías de la información y la comunicación (TIC) en la gestión empresarial de sectores productivos no formales, Intersedes, 14(27), 1-15 (2013)

Toudert, D., La brecha digital en los contextos de marginación socio-territorial de las localidades mexicanas de más de 2500 habitantes: Exploración y discusión. Comunicación y Sociedad, (19) 3, 53-180 (2013) 
